\title{
Existence Results for a Riemann-Liouville-Type Fractional Multivalued Problem with Integral Boundary Conditions
}

\author{
Hamed H. Alsulami, ${ }^{1}$ Sotiris K. Ntouyas, ${ }^{2}$ and Bashir Ahmad ${ }^{1}$ \\ ${ }^{1}$ Mathematics Department, Faculty of Science, King Abdulaziz University, P.O. Box 80203, Jeddah 21589, Saudi Arabia \\ ${ }^{2}$ Department of Mathematics, University of Ioannina, 45110 Ioannina, Greece
}

Correspondence should be addressed to Hamed H. Alsulami; hamed9@hotmail.com

Received 12 December 2012; Revised 29 December 2012; Accepted 2 January 2013

Academic Editor: Gennaro Infante

Copyright (c) 2013 Hamed H. Alsulami et al. This is an open access article distributed under the Creative Commons Attribution License, which permits unrestricted use, distribution, and reproduction in any medium, provided the original work is properly cited.

We discuss the existence of solutions for a boundary value problem of Riemann-Liouville fractional differential inclusions of order $\alpha \in(2,3]$ with integral boundary conditions. We establish our results by applying the standard tools of fixed point theory for multivalued maps when the right-hand side of the inclusion has convex as well as nonconvex values. An illustrative example is also presented.

\section{Introduction}

In the last few decades, fractional calculus is found to be an effective modeling tool in many branches of physics, economics, and technical sciences [1-3]. A fractional-order differential operator is nonlocal in its character in contrast to its counterpart in classical calculus. It means that the future state of a dynamical system or process based on fractional-order derivative depends on both its current and past states. Thus, the application of fractional calculus in various materials and processes enables an investigator to study the complete behavior (ranging from past to current states) of such stuff. This is indeed an important feature that makes fractionalorder models more realistic and practical than the integerorder models and has accounted for the popularity of the subject. For some recent development on the topic, see [417] and the references therein.

Differential inclusions appear in the mathematical modeling of certain problems in economics, optimal control, and so forth and are widely studied by many authors. Examples and details can be found in a series of papers [18-23] and the references cited therein.

In this paper, we study the following boundary value problem:

$$
D_{0+}^{\alpha} x(t) \in F(t, x(t)), \quad 0<t<1,2<\alpha \leq 3,
$$

$$
\begin{gathered}
x(0)=x^{\prime}(0)=0, \\
x(1)=\int_{0}^{1} g(s) x(s) d s,
\end{gathered}
$$

where $D_{0+}^{\alpha}$ is the standard Riemann-Liouville fractional derivative of order $2<\alpha \leq 3, F:[0,1] \times \mathbb{R} \rightarrow \mathscr{P}(\mathbb{R})$ is a multivalued map, $\mathscr{P}(\mathbb{R})$ is the family of all subsets of $\mathbb{R}$, and $g$ is a continuous function.

Here we remark that the present work is motivated by a recent paper [17], where problem (1) is considered with $F$ as single valued and the results on existence and nonexistence of positive solutions are obtained.

The main tools of our study include nonlinear alternative of Leray-Schauder type, a selection theorem due to Bressan and Colombo for lower semicontinuous multivalued maps, and Covitz and Nadler's fixed point theorem for contraction multivalued maps. The application of these results is new in the framework of the problem at hand. We recall some preliminaries in Section 2 while the main results are presented in Section 3.

\section{Preliminaries}

2.1. Fractional Calculus. Let us recall some basic definitions of fractional calculus $[1,2]$. 
Definition 1. The Riemann-Liouville derivative of fractional order $q$ is defined as

$$
\begin{array}{r}
D_{0+}^{q} g(t)=\frac{1}{\Gamma(n-q)}\left(\frac{d}{d t}\right)^{n} \int_{0}^{t}(t-s)^{n-q-1} g(s) d s, \\
n-1<q<n, \quad n=[q]+1,
\end{array}
$$

provided the integral exists, where $[q]$ denotes the integer part of the real number $q$.

Definition 2. The Riemann-Liouville fractional integral of order $q$ is defined as

$$
I_{0+}^{q} g(t)=\frac{1}{\Gamma(q)} \int_{0}^{t} \frac{g(s)}{(t-s)^{1-q}} d s, \quad q>0,
$$

provided the integral exists.

Definition 3. A function $x \in A C^{2}([0,1], \mathbb{R})$ is called a solution of problem $(1)$ if there exists a function $v \in L^{1}([0,1], \mathbb{R})$ with $v(t) \in F(t, x(t))$, a.e. $[0,1]$ such that $D^{\alpha} x(t)=v(t)$, a.e. $[0,1]$ and $x(0)=x^{\prime}(0)=0, x(1)=\int_{0}^{1} g(t) x(t) d t$.

Lemma 4 (see [17]). Given $y \in L([0,1], \mathbb{R})$, then the unique solution of the problem

$$
\begin{gathered}
D^{\alpha} x(t)+y(t)=0, \quad 0<t<1,2<\alpha \leq 3, \\
x(0)=x^{\prime}(0)=0, \\
x(1)=\int_{0}^{1} g(t) x(t) d t
\end{gathered}
$$

is given by

$$
x(t)=\int_{0}^{1} G(t, s) y(s) d s
$$

where

$$
\begin{gathered}
G(t, s)=G_{1}(t, s)+G_{2}(t, s), \\
G_{1}(t, s)=\frac{1}{\Gamma(\alpha)} \begin{cases}{[t(1-s)]^{\alpha-1},} & 0 \leq t \leq s \leq 1, \\
{[t(1-s)]^{\alpha-1}-(t-s)^{\alpha-1},} & 0 \leq s \leq t \leq 1,\end{cases} \\
G_{2}(t, s)=\frac{t^{\alpha-1}}{1-\sigma} \int_{0}^{1} G_{1}(\tau, s) g(\tau) d \tau \\
\sigma=\int_{0}^{1} s^{\alpha-1} g(s) d s .
\end{gathered}
$$

Lemma 5 (see [17]). The functions $G_{1}(t, s), G(t, s)$ have the following properties:

(i) $G_{1}(t, s) \geq 0$, for all $t, s \in[0,1]$;

(ii) $G_{1}(t, s) \leq\left((\alpha-1) s(1-s)^{\alpha-1}\right) / \Gamma(\alpha)$ for all $t, s \in[0,1]$;

(iii) $G(t, s) \leq\left(\left((\alpha-1) s(1-s)^{\alpha-1}\right) / \Gamma(\alpha)\right)(1+(1 /(1-$ $\left.\sigma)) \int_{0}^{1} g(\tau) d \tau\right)$ for all $t, s \in[0,1]$.
2.2. Basic Concepts of Multivalued Maps. Let $(\mathscr{X},\|\cdot\|)$ be a normed space and let $H: \mathscr{X} \rightarrow \mathscr{P}(\mathscr{X})$ be a multivalued map. $H$ is said to be

(i) convex (closed) valued if $H(x)$ is convex (closed) for all $x \in \mathscr{X}$;

(ii) bounded on bounded sets if $H(\mathbb{B})=\cup_{x \in \mathbb{B}} H(x)$ is bounded in $\mathscr{X}$ for all $\mathbb{B} \in \mathscr{P}_{b}(\mathscr{X})$, where $\mathscr{P}_{b}(\mathscr{X})=$ $\{\mathscr{A} \in \mathscr{P}(\mathscr{X}): \mathscr{A}$ is bounded $\}$, (i.e., $\sup _{x \in \mathbb{B}}\{\sup \{|w|$ : $w \in H(x)\}\}<\infty)$;

(iii) upper semicontinuous (u.s.c.) on $\mathscr{X}$ if the set $H\left(a_{0}\right)$ is a nonempty closed subset of $\mathscr{X}$ for each $a_{0} \in \mathscr{X}$ and if for each open set $M$ of $\mathscr{X}$ containing $H\left(a_{0}\right)$ there exists an open neighborhood $M_{0}$ of $a_{0}$ such that $H\left(M_{0}\right) \subseteq M$;

(iv) completely continuous if $H(\mathbb{B})$ is relatively compact for every $\mathbb{B} \in \mathscr{P}_{b}(\mathscr{X})$.

Definition 6. If the multivalued map $H$ is completely continuous with nonempty compact values, then $H$ is u.s.c. if and only if $H$ has a closed graph; that is, $w_{n} \in H\left(x_{n}\right)$ imply that $w_{*} \in H\left(x_{*}\right)$ when $x_{n} \rightarrow x_{*}, w_{n} \rightarrow w_{*}$.

Definition 7. $H$ has a fixed point if there is $x \in \mathscr{X}$ such that $x \in H(x)$. The fixed point set of the multivalued operator $H$ will be denoted by Fix $H$.

Definition 8. Let $\mathscr{P}_{c l}(\mathbb{R})=\{W \in \mathscr{P}(\mathbb{R}): W$ is closed $\}$. A multivalued map $H:[0 ; 1] \rightarrow \mathscr{P}_{c l}(\mathbb{R})$ is said to be measurable if the function

$$
t \longmapsto d(y, H(t))=\inf \{|y-z|: z \in H(t)\}
$$

is measurable for every $y \in \mathbb{R}$.

In the sequel, by $C([0,1])$ we mean a Banach space of continuous functions from $[0,1]$ into $\mathbb{R}$ with the norm $\|x\|=$ $\sup _{t \in[0,1]}|x(t)|$ whereas $L^{1}([0,1], \mathbb{R})$ is the Banach space of measurable functions $x:[0,1] \rightarrow \mathbb{R}$ which are Lebesgue integrable and normed by $\|x\|_{L^{1}}=\int_{0}^{1}|x(t)| d t$.

Definition 9. A multivalued map $\mathscr{H}:[0,1] \times \mathbb{R} \rightarrow \mathscr{P}(\mathbb{R})$ is said to be Carathéodory if the map $t \mapsto \mathscr{H}(t, x)$ is measurable for each $x \in \mathbb{R}$ and the map $x \mapsto \mathscr{H}(t, x)$ is upper semicontinuous for almost all $t \in[0,1]$. Further, a Carathéodory function $\mathscr{H}$ is called $L^{1}$-Carathéodory if for each $\alpha>0$, there exists $\varphi_{\alpha} \in L^{1}\left([0,1], \mathbb{R}^{+}\right)$such that $\|\mathscr{H}(t, x)\|=\sup \{|v|: v \in \mathscr{H}(t, x)\} \leq \varphi_{\alpha}(t)$ for all $\|x\| \leq \alpha$ and for a. e. $t \in[0,1]$.

Definition 10. For each $w \in C([0,1], \mathbb{R})$, the set of selections of $\mathscr{H}$ is defined by

$$
\begin{array}{r}
S_{\mathscr{H}, w}:=\left\{v \in L^{1}([0,1], \mathbb{R}): v(t) \in \mathscr{H}(t, w(t))\right. \\
\text { for a.e. } t \in[0,1]\} .
\end{array}
$$

Definition 11. For a nonempty closed subset $V$ of a Banach space $E$, let $\mathscr{H}_{c}: V \rightarrow \mathscr{P}(E)$ be a nonempty multivalued 
operator with closed values. We call $\mathscr{H}_{c}$ to be lower semicontinuous (1.s.c.) if the set $\left\{v \in V: \mathscr{H}_{c}(v) \cap B \neq \emptyset\right\}$ is open for any open set $U$ in $E$.

Definition 12. A subset $\mathscr{A}$ of $[0,1] \times \mathbb{R}$ is $\mathscr{L} \otimes \mathscr{B}$ measurable if $\mathscr{A}$ belongs to the $\sigma$-algebra generated by all sets of the form $\mathscr{J} \times \mathscr{D}$, where $\mathscr{J}$ is Lebesgue measurable in $[0,1]$ and $\mathscr{D}$ is Borel measurable in $\mathbb{R}$.

Definition 13. A subset $\mathscr{A}_{d}$ of $L^{1}([0,1], \mathbb{R})$ is said be decomposable if for all $a, b \in \mathscr{A}_{d}$ and measurable $\mathscr{K} \subset[0,1]$, the function $a \phi_{\mathscr{K}}+b \phi_{[0,1] \backslash \mathscr{K}} \in \mathscr{A}_{d}$, where $\phi_{\mathscr{K}}$ stands for the characteristic function of $\mathscr{K}$.

Definition 14. A multivalued operator $\mathscr{H}_{b c}: W \rightarrow \mathscr{P}\left(L^{1}([0,1]\right.$, $\mathbb{R})$ ) has a property $(\mathrm{BC})$ if $\mathscr{H}_{b c}$ is lower semi-continuous (l.s.c.) and has nonempty closed and decomposable values, where $W$ is a separable metric space.

Definition 15. Let $F:[0,1] \times \mathbb{R} \rightarrow \mathscr{P}(\mathbb{R})$ be a multivalued map with nonempty compact values. We say that $F$ is of lower semi-continuous type (l.s.c. type) if its associated Nemytskii operator $\mathscr{F}_{N}$ is lower semi-continuous and has nonempty closed and decomposable values, where

$$
\begin{aligned}
\mathscr{F}_{N}(x)= & \left\{u \in L^{1}([0,1], \mathbb{R}):\right. \\
& u(t) \in F(t, x(t)) \quad \text { for a.e. } t \in[0,1]\} .
\end{aligned}
$$

Definition 16. Let $(\mathscr{X}, d)$ be a metric space induced from the normed space $(\mathscr{X} ;\|\cdot\|)$ and let $\mathscr{M}_{d}: \mathscr{P}(\mathscr{X}) \times \mathscr{P}(\mathscr{X}) \rightarrow$ $\mathbb{R} \cup\{\infty\}$ be defined by

$$
\mathscr{M}_{d}(A, B)=\max \left\{\sup _{a \in A} d(a, B), \sup _{b \in B} d(A, b)\right\},
$$

where $d(A, b)=\inf _{a \in A} d(a ; b)$ and $d(a, B)=\inf _{b \in B} d(a ; b)$. Then $\left(\mathscr{P}_{b, c l}(\mathscr{X}), \mathscr{M}_{d}\right)$ is a metric space and $\left(\mathscr{P}_{c l}(\mathscr{X}), M_{d}\right)$ is a generalized metric space (see [24]).

Definition 17. A multivalued operator $\mathscr{H}: \mathscr{X} \rightarrow \mathscr{P}_{c l}(\mathscr{X})$ is called $\gamma$-Lipschitz if and only if there exists $\gamma>0$ such that

$$
\mathscr{M}_{d}(N(x), N(y)) \leq \gamma d(x, y) \text { for each } x, y \in \mathscr{X} \text {, }
$$

and a contraction if and only if it is $\gamma$-Lipschitz with $\gamma<1$.

For further details on multi-valued maps, we refer the reader to the books $[25,26]$.

\section{Existence Results for the Multivalued Problem}

In this section, we present some existence results for the problem (1). Our first result deals with the case when $F$ is Carathéodory. We make use of the following known results to establish the proof.
Lemma 18 (see [27]). Let $X$ be a Banach space. Let $F:[0, T] \times$ $\mathbb{R} \rightarrow \mathscr{P}_{c p, c}(X)$ be an $L^{1}$-Carathéodory multivalued map with compact and convex values and let $\theta$ be a linear continuous mapping from $L^{1}([0,1], X)$ to $C([0,1], X)$. Then the operator

$$
\begin{gathered}
\theta \circ S_{F}: C([0,1], X) \longrightarrow \mathscr{P}_{c p, c}(C([0,1], X)), \\
x \longmapsto\left(\theta \circ S_{F}\right)(x)=\theta\left(S_{F, x}\right)
\end{gathered}
$$

is a closed graph operator in $C([0,1], X) \times C([0,1], X)$.

Lemma 19 (nonlinear alternative for Kakutani maps [28]). Let $E$ be a Banach space, $C$ a closed convex subset of $E, U$ an open subset of $C$, and $0 \in U$. Suppose that $F: \bar{U} \rightarrow P_{c, c v}(C)$ is an upper semicontinuous compact map; here $P_{c, c v}(C)$ denotes the family of nonempty, compact convex subsets of $C$. Then either

(i) F has a fixed point in $\bar{U}$, or

(ii) there is a $u \in \partial U$ and $\lambda \in(0,1)$ with $u \in \lambda F(u)$.

Theorem 20. Assume that

$\left(\mathrm{H}_{1}\right) F:[0,1] \times \mathbb{R} \rightarrow \mathscr{P}(\mathbb{R})$ is Carathéodory and has nonempty compact and convex values;

$\left(\mathrm{H}_{2}\right)$ there exists a continuous nondecreasing function $\psi$ : $[0, \infty) \rightarrow(0, \infty)$ and a function $p \in L^{1}\left([0,1], \mathbb{R}^{+}\right)$ such that

$$
\begin{aligned}
\|F(t, x)\|_{\mathscr{P}} & :=\sup \{|y|: y \in F(t, x)\} \\
& \leq p(t) \psi(\|x\|) \text { for each }(t, x) \in[0,1] \times \mathbb{R} ;
\end{aligned}
$$

$\left(\mathrm{H}_{3}\right)$ there exists a constant $\widetilde{M}>0$ such that

$$
\begin{gathered}
\widetilde{M}\left(\psi(\widetilde{M}) \frac{(\alpha-1)}{\Gamma(\alpha)}\left(1+\frac{1}{1-\sigma} \int_{0}^{1} g(s) d s\right)\right. \\
\left.\times \int_{0}^{1} s(1-s)^{\alpha-1} p(s) d s\right)^{-1}>1 .
\end{gathered}
$$

Then the boundary value problem (1) has at least one solution on $[0,1]$.

Proof. In view of Lemma 4, we define an operator $\mathbb{Q}$ : $C([0,1], \mathbb{R}) \rightarrow \mathscr{P}(C([0,1], \mathbb{R}))$ by

$$
\mathcal{Q}(x)=\left\{h \in C([0,1], \mathbb{R}): h(t)=\int_{0}^{1} G(t, s) v(s) d s, v \in S_{F, x}\right\}
$$

and show that it satisfies the hypotheses of Lemma 19. Since $S_{F, x}$ is convex ( $F$ has convex values), therefore, it can be easily shown that $Q$ is convex for each $x \in C([0,1], \mathbb{R})$.

As a next step, we prove that $Q$ maps the bounded sets (balls) into bounded sets in $C([0,1], \mathbb{R})$. For a positive number $\xi$, let $B_{\xi}=\{x \in C([0,1], \mathbb{R}):\|x\| \leq \xi\}$ be a bounded ball in $C([0,1], \mathbb{R})$. Then, for each $h \in Q(x), x \in B_{\xi}$, there exists $v \in S_{F, x}$ such that

$$
h(t)=\int_{0}^{1} G(t, s) v(s) d s
$$


Then, for $t \in[0,1]$, we have

$$
\begin{aligned}
|h(t)| \leq & \int_{0}^{1} G(t, s)|v(s)| d s \\
\leq & \psi(\|x\|) \frac{(\alpha-1)}{\Gamma(\alpha)}\left(1+\frac{1}{1-\sigma} \int_{0}^{1} g(s) d s\right) \\
& \times \int_{0}^{1} s(1-s)^{\alpha-1} p(s) d s \\
\leq & \psi(\xi) \frac{(\alpha-1)}{\Gamma(\alpha)}\left(1+\frac{1}{1-\sigma} \int_{0}^{1} g(s) d s\right) \\
& \times \int_{0}^{1} s(1-s)^{\alpha-1} p(s) d s .
\end{aligned}
$$

Thus,

$$
\begin{aligned}
\|h\| \leq & \psi(\xi) \frac{(\alpha-1)}{\Gamma(\alpha)}\left(1+\frac{1}{1-\sigma} \int_{0}^{1} g(s) d s\right) \\
& \times \int_{0}^{1} s(1-s)^{\alpha-1} p(s) d s .
\end{aligned}
$$

Now we show that $Q$ maps the bounded sets into equicontinuous sets of $C([0,1], \mathbb{R})$.

Let $t^{\prime}, t^{\prime \prime} \in[0,1]$ with $t^{\prime}<t^{\prime \prime}$ and $x \in B_{\xi}$. For each $h \in \mathbb{Q}$, we obtain

$$
\begin{aligned}
\left|h\left(t^{\prime \prime}\right)-h\left(t^{\prime}\right)\right| & \leq \int_{0}^{1}\left|G\left(t^{\prime}, s\right)-G\left(t^{\prime \prime}, s\right) \| v(s)\right| d s \\
& \leq \int_{0}^{1}\left|G\left(t^{\prime}, s\right)-G\left(t^{\prime \prime}, s\right)\right| p(s) \psi\|x\| d s \\
& \leq \psi(\xi) \int_{0}^{1}\left|G\left(t^{\prime}, s\right)-G\left(t^{\prime \prime}, s\right)\right| p(s) d s .
\end{aligned}
$$

Obviously the right-hand side of the above inequality tends to zero independently of $x \in B_{\xi}$ as $t^{\prime \prime}-t^{\prime} \rightarrow 0$. As $Q$ satisfies the above three assumptions, therefore, by the Ascoli-Arzelá theorem, it follows that $\mathbb{Q}: C([0,1], \mathbb{R}) \rightarrow \mathscr{P}(C([0,1], \mathbb{R}))$ is completely continuous.

In our next step, we show that $\mathbb{Q}$ has a closed graph. Let $x_{n} \rightarrow x_{*}, h_{n} \in \mathbb{Q}\left(x_{n}\right)$, and $h_{n} \rightarrow h_{*}$. Then we need to show that $h_{*} \in \mathbb{Q}\left(x_{*}\right)$. Associated with $h_{n} \in \mathbb{Q}\left(x_{n}\right)$, there exists $v_{n} \in S_{F, x_{n}}$ such that for each $t \in[0,1]$,

$$
h_{n}(t)=\int_{0}^{1} G(t, s) v_{n}(s) d s .
$$

Thus, it suffices to show that there exists $v_{*} \in S_{F, x_{*}}$ such that for each $t \in[0,1]$,

$$
h_{*}(t)=\int_{0}^{1} G(t, s) v_{*}(s) d s .
$$

Define a linear operator $\varphi: L^{1}([0,1], \mathbb{R}) \rightarrow C([0,1], \mathbb{R})$ as

$$
f \longmapsto \varphi(v)(t)=\int_{0}^{1} G(t, s) v(s) d s .
$$

Notice that

$$
\begin{aligned}
& \left\|h_{n}(t)-h_{*}(t)\right\| \\
& \quad=\left\|\int_{0}^{1} G(t, s)\left(v_{n}(s)-v_{*}(s)\right) d s\right\| \longrightarrow 0, \quad \text { as } n \longrightarrow \infty .
\end{aligned}
$$

Thus, it follows from Lemma 18 that $\varphi \circ S_{F}$ is a closed graph operator. Further, we have $h_{n}(t) \in \varphi\left(S_{F, x_{n}}\right)$, since $x_{n} \rightarrow x_{*}$. Thus, for some $v_{*} \in S_{F, x_{*}}$, we have

$$
h_{*}(t)=\int_{0}^{1} G(t, s) v_{*}(s) d s .
$$

In the last step, we show that there exists an open set $\mathscr{V} \subseteq$ $C([0,1], \mathbb{R})$ with $x \notin Q Q(x)$ for any $\mu \in(0,1)$ and all $x \in$ $\partial \mathscr{V}$. Let $\mu \in(0,1)$ and $x \in \mu Q(x)$. Then there exists $v \in$ $L^{1}([0,1], \mathbb{R})$ with $v \in S_{F, x}$ such that for $t \in[0,1]$, we have

$$
x(t)=\mu \int_{0}^{1} G(t, s) v(s) d s,
$$

and using the computations used in the second step, we obtain

$$
\begin{aligned}
|x(t)| \leq & \psi(\|x\|) \frac{(\alpha-1)}{\Gamma(\alpha)}\left(1+\frac{1}{1-\sigma} \int_{0}^{1} g(s) d s\right) \\
& \times \int_{0}^{1} s(1-s)^{\alpha-1} p(s) d s .
\end{aligned}
$$

In consequence, we have

$$
\begin{gathered}
\|x\|\left(\psi(\|x\|) \frac{(\alpha-1)}{\Gamma(\alpha)}\left(1+\frac{1}{1-\sigma} \int_{0}^{1} g(s) d s\right)\right. \\
\left.\times \int_{0}^{1} s(1-s)^{\alpha-1} p(s) d s\right)^{-1} \leq 1 .
\end{gathered}
$$

By the assumption $\left(\mathrm{H}_{3}\right)$, there exists $\widetilde{M}$ such that $\|x\| \neq \widetilde{M}$. Let us set

$$
\mathscr{V}=\{x \in C([0,1], \mathbb{R}):\|x\|<\widetilde{M}\}
$$

Observe that the operator $Q: \overline{\mathscr{V}} \rightarrow \mathscr{P}(C([0,1], \mathbb{R}))$ is upper semicontinuous and completely continuous. From the choice of $\mathscr{V}$, there is no $x \in \partial \mathscr{V}$ such that $x \in \mu Q(x)$ for some $\mu \in(0,1)$. Consequently, by Lemma 19 , we have that $\mathbb{Q}$ has a fixed point $x \in \overline{\mathscr{V}}$ which is a solution of the problem (1). This completes the proof.

Example 21. Consider the following boundary value problem:

$$
\begin{gathered}
{ }^{c} D^{5 / 2} x(t) \in F(t, x(t)), \quad t \in[0,1], \\
x(0)=x^{\prime}(0)=0, \\
x(1)=\int_{0}^{1} \sqrt{t} x(t) d t,
\end{gathered}
$$


where $F:[0,1] \times \mathbb{R} \rightarrow \mathscr{P}(\mathbb{R})$ is a multivalued map given by

$$
x \rightarrow F(t, x)=\left[\frac{t|x|^{3}}{\left(|x|^{3}+3\right)}, \frac{t|x|}{2(|x|+1)}\right] .
$$

For $f \in F$, we have

$$
|f| \leq \max \left(\frac{t|x|^{3}}{\left(|x|^{3}+3\right)}, \frac{t|x|}{2(|x|+1)}\right) \leq \sqrt{t}, \quad x \in \mathbb{R} .
$$

Here $\sigma=\int_{0}^{1} s^{\alpha-1} g(s) d s=\int_{0}^{1} s^{3 / 2} s^{1 / 2} d s=(1 / 3)<1$, $\|F(t, x)\|_{\mathscr{P}}:=\sup \{|y|: y \in F(t, x)\} \leq t=p(t) \psi(\|x\|), x \in \mathbb{R}$, with $p(t)=t, \psi(\|x\|)=1$, and $\int_{0}^{1} s(1-s)^{3 / 2} s d s=16 / 315$. Using the given values in the condition $\left(\mathrm{H}_{3}\right)$ :

$$
\begin{gathered}
M\left(\psi(M) \frac{(\alpha-1)}{\Gamma(\alpha)}\left(1+\frac{1}{1-\sigma} \int_{0}^{1} g(s) d s\right)\right. \\
\left.\times \int_{0}^{1} s(1-s)^{\alpha-1} p(s) d s\right)^{-1}>1
\end{gathered}
$$

we find that

$$
M>\frac{96}{315 \sqrt{\pi}} .
$$

Clearly, all the conditions of Theorem 20 are satisfied. Hence, the conclusion of Theorem 20 applies to the problem (31).

In our next result, we assume that $F$ is not necessarily convex valued. We complete the proof of this result by applying the nonlinear alternative of Leray-Schauder type together with the selection theorem of Bressan and Colombo [29] for lower semi-continuous maps with decomposable values, which is stated below.

Lemma 22 (see $[29,30])$. Let $Y$ be a separable metric space and let $N: Y \rightarrow \mathscr{P}\left(L^{1}([0,1], \mathbb{R})\right)$ be a multivalued operator satisfying the property $(B C)$. Then $N$ has a continuous selection; that is, there exists a continuous function (singlevalued) $g: Y \rightarrow L^{1}([0,1], \mathbb{R})$ such that $g(x) \in N(x)$ for every $x \in Y$.

Theorem 23. Suppose that $\left(\mathrm{H}_{2}\right)$ and $\left(\mathrm{H}_{3}\right)$ hold. In addition, we assume the following condition:

$\left(\mathrm{H}_{4}\right) F:[0,1] \times \mathbb{R} \rightarrow \mathscr{P}(\mathbb{R})$ is a nonempty compact-valued multivalued map such that

(a) $(t, x) \mapsto F(t, x)$ is $\mathscr{L} \otimes \mathscr{B}$ measurable,

(b) $x \mapsto F(t, x)$ is lower semicontinuous for each $t \in$ $[0,1]$.

Then the problem (1) has at least one solution on $[0,1]$.

Proof. Observe that the assumptions $\left(\mathrm{H}_{2}\right)$ and $\left(\mathrm{H}_{4}\right)$ imply that $F$ is of l.s.c. type. Then, by Lemma 22 , there exists a continuous function $v: A C^{2}([0,1], \mathbb{R}) \rightarrow L^{1}([0,1], \mathbb{R})$ such that $v(x) \in \mathscr{F}(x)$ for all $x \in C([0,1], \mathbb{R})$.
Let us consider the problem

$$
\begin{gathered}
D^{\alpha} x(t)=v(x(t)), \quad t \in[0,1], \\
x(0)=x^{\prime}(0)=0, \\
x(1)=\int_{0}^{1} g(t) x(t) d t .
\end{gathered}
$$

One can note that if $x \in A C^{2}([0,1], \mathbb{R})$ is a solution of (36), then $x$ is a solution to the problem (1). To convert the problem (36) to a fixed point problem, we define an operator $\bar{Q}$ as

$$
\bar{Q} x(t)=\int_{0}^{1} G(t, s) v(x(s)) d s .
$$

It is easy to show that the operator $\bar{Q}$ is continuous and completely continuous. The rest of the proof is similar to that of Theorem 20. So we omit it. This completes the proof.

Finally we show the existence of solutions for the problem (1) with a nonconvex valued right-hand side by applying a fixed point theorem for multivalued maps according to Covitz and Nadler [31].

Lemma 24 ([31]). Let $(X, d)$ be a complete metric space. If $N$ : $X \rightarrow \mathscr{P}_{c l}(X)$ is a contraction, then Fix $N \neq \emptyset$.

Theorem 25. Assume that

$\left(\mathrm{H}_{5}\right) F:[0,1] \times \mathbb{R} \rightarrow \mathscr{P}_{c p}(\mathbb{R})$ is such that $F(\cdot, x):[0,1] \rightarrow$ $\mathscr{P}_{c p}(\mathbb{R})$ is measurable for each $x \in \mathbb{R}$;

$\left(\mathrm{H}_{6}\right) \mathscr{M}_{d}(F(t, x), F(t, y)) \leq m(t)|x-y|$ for almost all $t \in$ $[0,1]$ and $x, y \in \mathbb{R}$ with $m \in L^{1}\left([0,1], \mathbb{R}^{+}\right)$and $d(0, F(t, 0)) \leq m(t)$ for almost all $t \in[0,1]$.

Then the boundary value problem (1) has at least one solution on $[0,1]$ if

$$
\frac{(\alpha-1)}{\Gamma(\alpha)}\left(1+\frac{1}{1-\sigma} \int_{0}^{1} g(s) d s\right) \int_{0}^{1} s(1-s)^{\alpha-1} m(s) d s<1 .
$$

Proof. By the assumption $\left(\mathrm{H}_{5}\right)$, it follows that the set $S_{F, x}$ is nonempty for each $x \in C([0,1], \mathbb{R})$. So $F$ has a measurable selection (see [32, Theorem III.6]). Now it will be shown that the operator $Q$ defined by (17) satisfies the hypotheses of Lemma 24. To show that $Q(x) \in \mathscr{P}_{c l}((C[0,1], \mathbb{R}))$ for each $x \in C([0,1], \mathbb{R})$, let $\left\{u_{n}\right\}_{n \geq 0} \in \mathbb{Q}(x)$ be such that $u_{n} \rightarrow u(n \rightarrow$ $\infty)$ in $C([0,1], \mathbb{R})$. Then $u \in C([0,1], \mathbb{R})$ and there exists $v_{n} \in S_{F, x_{n}}$ such that, for each $t \in[0,1]$,

$$
u_{n}(t)=\int_{0}^{1} G(t, s) v_{n}(s) d s .
$$

As $F$ has compact values, we pass onto a subsequence (if necessary) to obtain that $v_{n}$ converges to $v$ in $L^{1}([0,1], \mathbb{R})$. Thus, $v \in S_{F, x}$ and for each $t \in[0,1]$, we have

$$
v_{n}(t) \longrightarrow v(t)=\int_{0}^{1} G(t, s) v(s) d s .
$$

Hence, $u \in \mathbb{Q}$. 
Next, we show that there exists $\delta<1$ such that

$$
\begin{aligned}
& \mathscr{M}_{d}(\mathbb{Q}(x), Q(y)) \\
& \quad \leq \delta\|x-y\| \text { for each } x, y \in A C^{2}([0,1], \mathbb{R}) .
\end{aligned}
$$

Let $x, y \in A C^{2}([0,1], \mathbb{R})$ and $h_{1} \in \mathbb{Q}(x)$. Then there exists $v_{1}(t) \in F(t, x(t))$ such that, for each $t \in[0,1]$,

$$
h_{1}(t)=\int_{0}^{1} G(t, s) v_{1}(s) d s .
$$

By $\left(\mathrm{H}_{6}\right)$, we have that

$$
\mathscr{M}_{d}(F(t, x), F(t, y)) \leq m(t)|x(t)-y(t)| .
$$

So, there exists $w \in F(t, y(t))$ such that

$$
\left|v_{1}(t)-w\right| \leq m(t)|x(t)-y(t)|, \quad t \in[0,1] .
$$

Define $\mathscr{W}:[0,1] \rightarrow \mathscr{P}(\mathbb{R})$ by

$$
\mathscr{W}(t)=\left\{w \in \mathbb{R}:\left|v_{1}(t)-w\right| \leq m(t)|x(t)-y(t)|\right\} .
$$

Since the multivalued operator $\mathscr{W}(t) \cap F(t, y(t))$ is measurable ([32, Proposition III.4]), there exists a function $v_{2}(t)$ which is a measurable selection for $\mathscr{W}$. So $v_{2}(t) \in F(t, y(t))$ and for each $t \in[0,1]$, we have that $\left|v_{1}(t)-v_{2}(t)\right| \leq m(t)|x(t)-y(t)|$.

For each $t \in[0,1]$, let us define

$$
h_{2}(t)=\int_{0}^{1} G(t, s) v_{2}(s) d s .
$$

Thus,

$$
\begin{aligned}
\left|h_{1}(t)-h_{2}(t)\right| \leq & \int_{0}^{1} G(t, s)\left|v_{1}(s)-v_{2}(s)\right| d s \\
\leq & \frac{(\alpha-1)}{\Gamma(\alpha)}\left(1+\frac{1}{1-\sigma} \int_{0}^{1} g(s) d s\right) \\
& \times \int_{0}^{1} s(1-s)^{\alpha-1} m(s) d s\left|v_{1}(s)-v_{2}(s)\right| d s .
\end{aligned}
$$

Hence,

$$
\begin{aligned}
\left\|h_{1}-h_{2}\right\| \leq & \frac{(\alpha-1)}{\Gamma(\alpha)}\left(1+\frac{1}{1-\sigma} \int_{0}^{1} g(s) d s\right) \\
& \times \int_{0}^{1} s(1-s)^{\alpha-1} m(s) d s\|x-y\| .
\end{aligned}
$$

Analogously, interchanging the roles of $x$ and $\bar{x}$, we obtain

$$
\begin{aligned}
\mathscr{M}_{d}(\mathcal{Q}(x), \mathcal{Q}(y)) \leq & \delta\|x-y\| \\
\leq & \left\{\frac{(\alpha-1)}{\Gamma(\alpha)}\left(1+\frac{1}{1-\sigma} \int_{0}^{1} g(s) d s\right)\right. \\
& \left.\times \int_{0}^{1} s(1-s)^{\alpha-1} m(s) d s\right\}\|x-y\| .
\end{aligned}
$$

Since Q is a contraction, it follows from Lemma 24 that $Q$ has a fixed point $x$ which is a solution of (1). This completes the proof.

\section{Acknowledgments}

The authors thank the editor and the reviewer for their constructive comments that led to the improvement of the paper. The research of $\mathrm{H}$. H. Alsulami and B. Ahmad was partially supported by Deanship of Scientific Research (DSR), King Abdulaziz University, Jeddah, Saudi Arabia.

\section{References}

[1] I. Podlubny, Fractional Differential Equations, vol. 198, Academic Press, San Diego, Calif, USA, 1999.

[2] A. A. Kilbas, H. M. Srivastava, and J. J. Trujillo, Theory and Applications of Fractional Differential Equations, vol. 204 of North-Holland Mathematics Studies, Elsevier Science, Amsterdam, The Netherlands, 2006.

[3] D. Baleanu, K. Diethelm, E. Scalas, and J. J. Trujillo, Fractional Calculus Models and Numerical Methods, vol. 3 of Series on Complexity, Nonlinearity and Chaos, World Scientific Publishing, Boston, Mass, USA, 2012.

[4] R. P. Agarwal, B. Andrade, and C. Cuevas, "Weighted pseudoalmost periodic solutions of a class of semilinear fractional differential equations," Nonlinear Analysis, vol. 11, no. 5, pp. 3532-3554, 2010.

[5] Z. Bai, "On positive solutions of a nonlocal fractional boundary value problem," Nonlinear Analysis, vol. 72, no. 2, pp. 916-924, 2010.

[6] K. Balachandran and J. J. Trujillo, "The nonlocal Cauchy problem for nonlinear fractional integrodifferential equations in Banach spaces," Nonlinear Analysis, vol. 72, no. 12, pp. 45874593, 2010.

[7] D. Băleanu, O. G. Mustafa, and D. O’Regan, "A Nagumolike uniqueness theorem for fractional differential equations," Journal of Physics A, vol. 44, no. 39, Article ID 392003, 6 pages, 2011.

[8] C. Cuevas, H. Soto, and A. Sepúlveda, "Almost periodic and pseudo-almost periodic solutions to fractional differential and integro-differential equations," Applied Mathematics and Computation, vol. 218, no. 5, pp. 1735-1745, 2011.

[9] R. P. Agarwal, M. Benchohra, and S. Hamani, "A survey on existence results for boundary value problems of nonlinear fractional differential equations and inclusions," Acta Applicandae Mathematicae, vol. 109, no. 3, pp. 973-1033, 2010.

[10] S. Bhalekar, V. Daftardar-Gejji, D. Baleanu, and R. Magin, "Fractional Bloch equation with delay," Computers \& Mathematics with Applications, vol. 61, no. 5, pp. 1355-1365, 2011.

[11] B. Ahmad and S. K. Ntouyas, "A four-point nonlocal integral boundary value problem for fractional differential equations of arbitrary order," Electronic Journal of Qualitative Theory of Differential Equations, no. 22, article 15, 2011.

[12] N. J. Ford and M. L. Morgado, "Fractional boundary value problems: analysis and numerical methods," Fractional Calculus and Applied Analysis, vol. 14, no. 4, pp. 554-567, 2011.

[13] F. T. Akyildiz, H. Bellout, K. Vajravelu, and R. A. Van Gorder, "Existence results for third order nonlinear boundary value problems arising in nano boundary layer fluid flows over stretching surfaces," Nonlinear Analysis, vol. 12, no. 6, pp. 2919-2930, 2011.

[14] B. Ahmad and J. J. Nieto, "Riemann-Liouville fractional integrodifferential equations with fractional nonlocal integral boundary conditions," Boundary Value Problems, vol. 36, 9 pages, 2011. 
[15] B. Ahmad and J. J. Nieto, "Sequential fractional differential equations with three-point boundary conditions," Computers \& Mathematics with Applications, vol. 64, no. 10, pp. 3046-3052, 2012.

[16] B. Ahmad, J. J. Nieto, A. Alsaedi, and M. El-Shahed, "A study of nonlinear Langevin equation involving two fractional orders in different intervals," Nonlinear Analysis: Real World Applications, vol. 13, no. 2, pp. 599-606, 2012.

[17] X. Zhao, C. Chai, and W. Ge, "Existence and nonexistence results for a class of fractional boundary value problems," Journal of Applied Mathematics and Computing, vol. 41, no. 1-2, pp. 17-31, 2013.

[18] J. Henderson and A. Ouahab, "Fractional functional differential inclusions with finite delay," Nonlinear Analysis, vol. 70, no. 5, pp. 2091-2105, 2009.

[19] G. Infante and P. Pietramala, "Perturbed Hammerstein integral inclusions with solutions that change sign," Commentationes Mathematicae Universitatis Carolinae, vol. 50, no. 4, pp. 591605, 2009.

[20] S. K. Ntouyas, "Neumann boundary value problems for impulsive differential inclusions," Electronic Journal of Qualitative Theory of Differential Equations, no. 22, 13 pages, 2009.

[21] B. Ahmad, "Existence results for fractional differential inclusions with separated boundary conditions," Bulletin of the Korean Mathematical Society, vol. 47, no. 4, pp. 805-813, 2010.

[22] B. Ahmad and S. K. Ntouyas, "Some existence results for boundary value problems of fractional differential inclusions with non-separated boundary conditions," Electronic Journal of Qualitative Theory of Differential Equations, no. 71, pp. 1-17, 2010.

[23] R. P. Agarwal and B. Ahmad, "Existence theory for anti-periodic boundary value problems of fractional differential equations and inclusions," Computers \& Mathematics with Applications, vol. 62, no. 3, pp. 1200-1214, 2011.

[24] M. Kisielewicz, Differential Inclusions and Optimal Control, Kluwer Academic, Dordrecht, The Netherlands, 1991.

[25] K. Deimling, Multivalued Differential Equations, vol. 1, Walter de Gruyter, Berlin, Germany, 1992.

[26] S. Hu and N. Papageorgiou, Handbook of Multivalued Analysis. I, Kluwer Academic Publishers, Dordrecht, The Netherlands, 1997.

[27] A. Lasota and Z. Opial, "An application of the Kakutani-Ky Fan theorem in the theory of ordinary differential equations," Bulletin de l'Académie Polonaise des Sciences, vol. 13, pp. 781-786, 1965.

[28] A. Granas and J. Dugundji, Fixed Point Theory, Springer, New York, NY, USA, 2005.

[29] A. Bressan and G. Colombo, "Extensions and selections of maps with decomposable values," Studia Mathematica, vol. 90, no. 1, pp. 69-86, 1988.

[30] M. Frigon, "Théorèemes d'existence de solutions d'inclusions différentielles," in Topological Methods in Differential Equations and Inclusions, A. Granas and M. Frigon, Eds., vol. 472 of NATO ASI Series C, pp. 51-87, Kluwer Academic Publishers, Dordrecht, The Netherlands, 1995.

[31] H. Covitz and S. B. Nadler Jr., "Multi-valued contraction mappings in generalized metric spaces," Israel Journal of Mathematics, vol. 8, pp. 5-11, 1970.

[32] C. Castaing and M. Valadier, Convex Analysis and Measurable Multifunctions, vol. 580 of Lecture Notes in Mathematics, Springer, Berlin, Germany, 1977. 


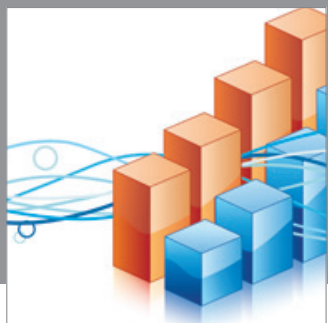

Advances in

Operations Research

mansans

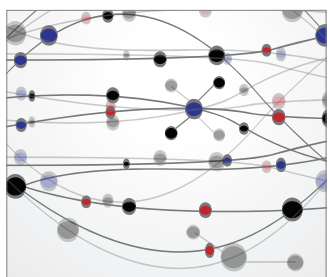

The Scientific World Journal
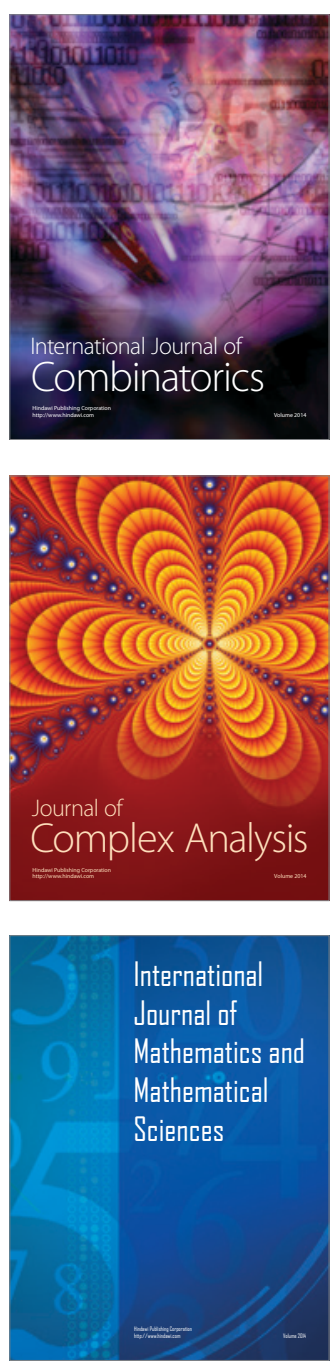
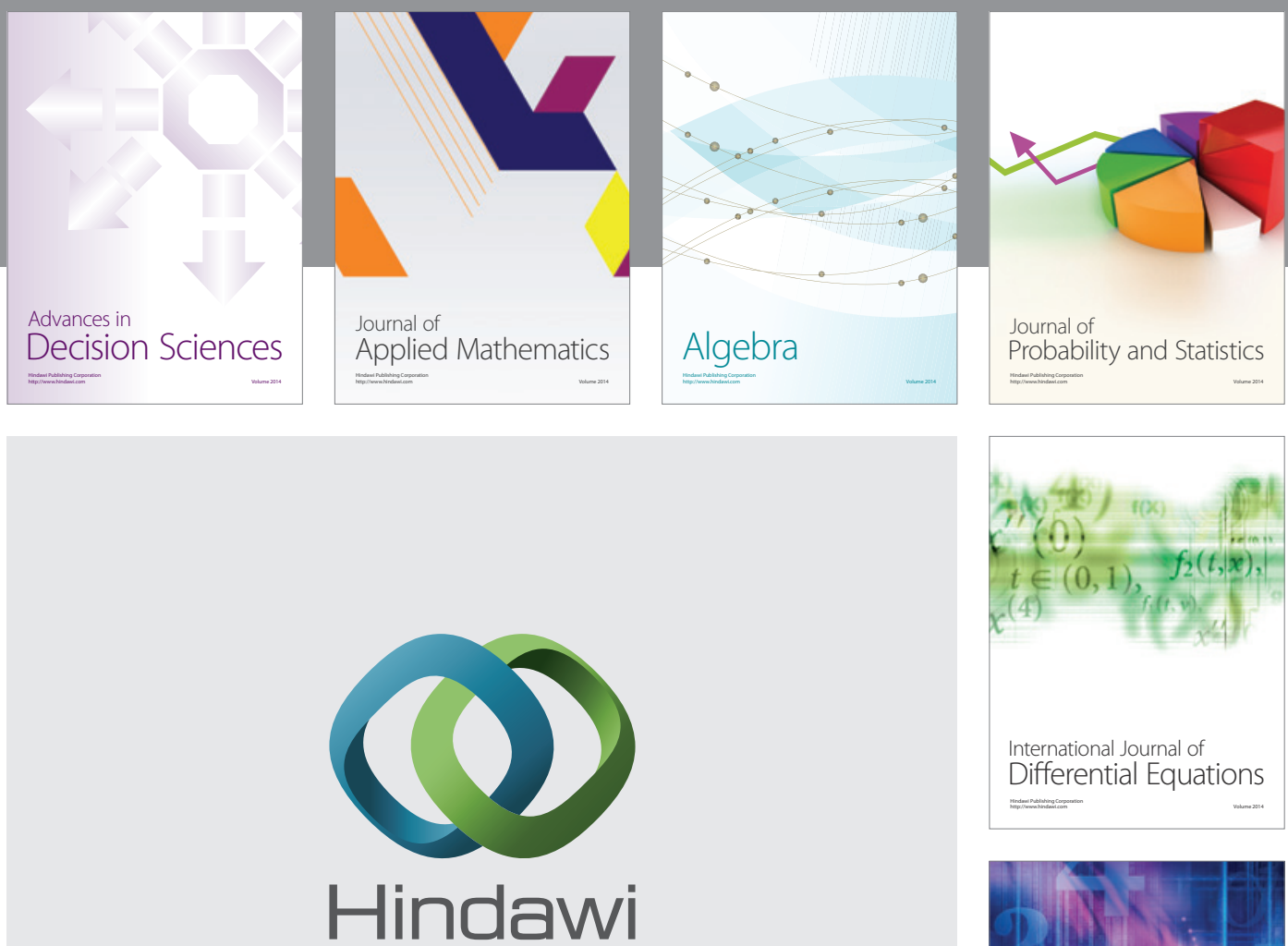

Submit your manuscripts at http://www.hindawi.com
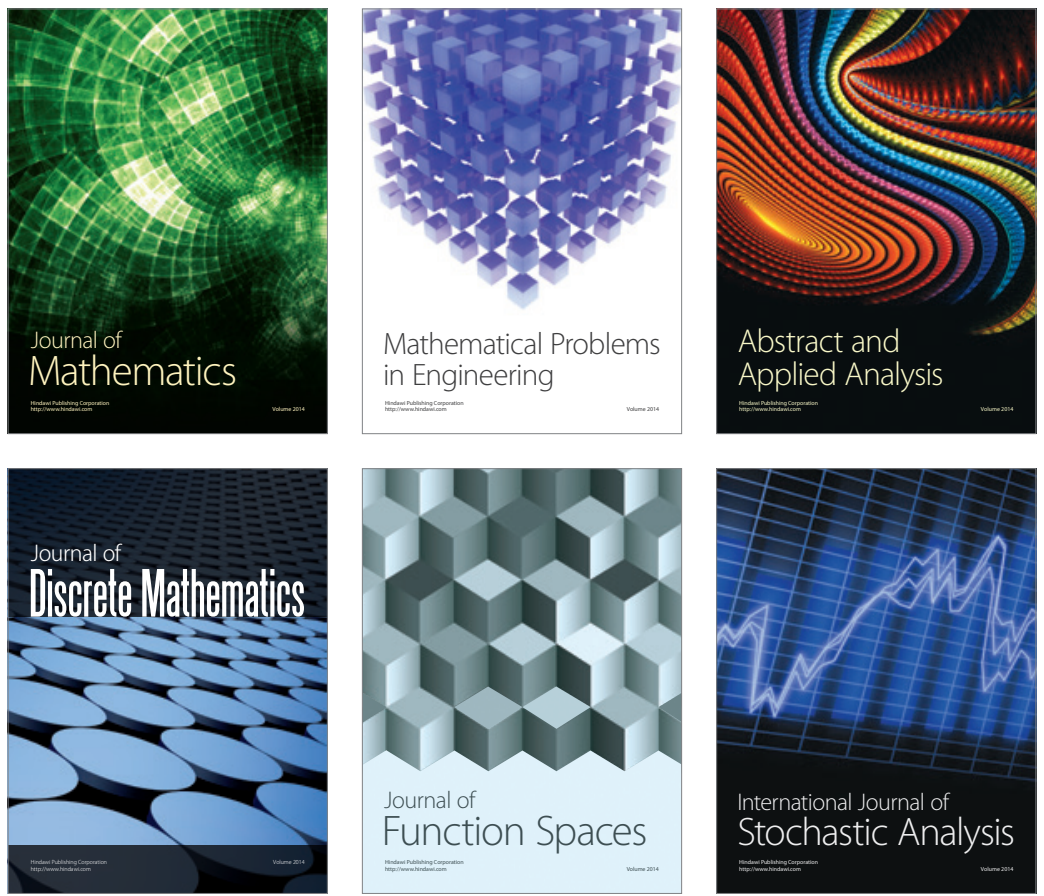

Journal of

Function Spaces

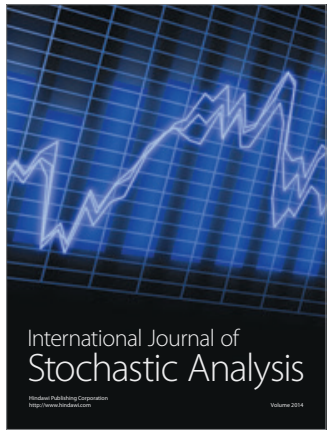

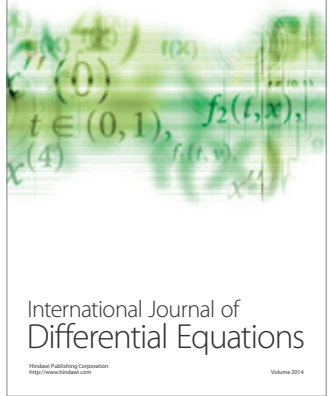
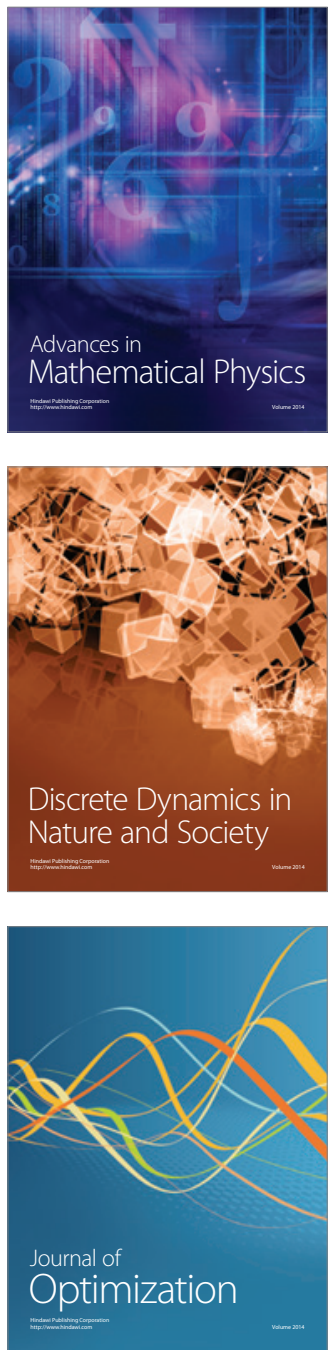